\title{
Contents
}

\section{Part I Theory}

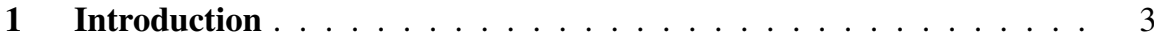

1.1 Background ................... 3

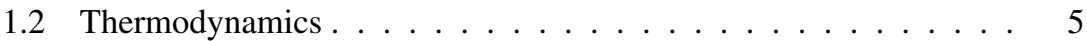

1.3 Hamiltonian Mechanics . . . . . . . . . . . . . . . . 6

1.4 Static Stability and Dynamic Stability . . . . . . . . . . . 6

1.5 Limit Cycles . . . . . . . . . . . . . . . . . . . 8

1.6 Information Theory . . . . . . . . . . . . . . . . . . 9

1.7 Chapter Summary . . . . . . . . . . . . . . . 11

2 Thermodynamics ......................... 13

2.1 Introduction . . . . . . . . . . . . . . . 13

2.2 First Law (Energy) . . . . . . . . . . . . . . . . . . . . . . . . . . . . . . . . . 13

2.3 Second Law (Stability/Entropy/Available Work) . . . . . . . . . . 14

2.4 Equilibrium Thermodynamics (Reversible/Irreversible Processes) . 18

2.5 Local Equilibrium (Nonequilibrium Thermodynamics; Energy,

Entropy, and Exergy Rate Equations) . . . . . . . . . . . . . . . . 19

2.6 Chapter Summary . . . . . . . . . . . . . . . . . . . . . . . . 21

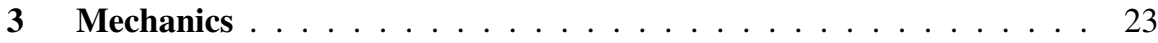

3.1 Introduction . . . . . . . . . . . . . 23

3.2 Work, Energy, and Power . . . . . . . . . . . . . . . 23

3.3 Energy Diagrams and Phase Planes . . . . . . . . . . . . . . 26

3.4 Hamiltonian Mechanics . . . . . . . . . . . . . . . . 27

3.5 Connections Between Thermodynamics and Hamiltonian

Mechanics . . . . . . . . . . . . . . . . . . 29

3.5.1 Conservative Mechanical Systems . . . . . . . . . . . . 29

3.5.2 Reversible Thermodynamic Systems . . . . . . . . . . . . 29

3.5.3 Irreversible Thermodynamic Systems . . . . . . . . . . . 30

3.5 .4 Connections ................. 30

3.6 Line Integrals and Limit Cycles . . . . . . . . . . . . . . 32 
3.6.1 Linear Limit Cycle . . . . . . . . . . . . . . . . . . . . . . 32

3.6.2 Nonlinear Limit Cycles . . . . . . . . . . . . . . . . . 39

3.6.3 Connection of Line Integrals and Limit Cycles to Thermodynamics ................ . . 51

3.7 Chapter Summary . . . . . . . . . . . . . . . . . . . 53

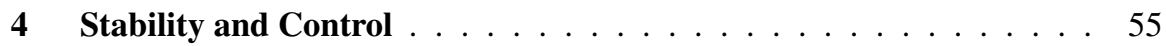

4.1 Introduction . . . . . . . . . . . . . . . . 55

4.2 Static Stability and Dynamic Stability . . . . . . . . . . 56

4.3 Eigenanalysis . . . . . . . . . . . . . . 60

4.3.1 Integral Feedback Is an Exergy Generator-Comparison to a Lag Stabilized System . . . . . . . . . . . . 63

4.3.2 Integral Feedback Is an Exergy Generator-Investigation by Exergy/Entropy Control Stability Boundary . . . . . . . 64

4.3.3 Integral Feedback Is an Exergy Generator-RouthHurwitz Stability Analysis . . . . . . . . . . . . . 65

4.3.4 PID Control Design Numerical Example . . . . . . . . . . 66

4.3.5 The Power Flow Principle of Stability for Nonlinear

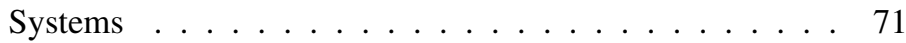

4.4 Lyapunov Analysis . . . . . . . . . . . . . . . . . . . 72

4.5 Energy Storage Surface and Power Flow: HSSPFC . . . . . . . 77

4.6 Chapter Summary . . . . . . . . . . . . . . . . . . . . 93

5 Advanced Control Design . . . . . . . . . . . . . . . . . . . 95

5.1 Introduction . . . . . . . . . . . . . . . . . . . . 95

5.2 Distributed Parameters/PDEs _ . . . . . . . . . . . . . . . . 95

5.3 Fractional Calculus . . . . . . . . . . . . . . . . . . 99

5.3.1 Sorting Power Terms: Generators, Storage, Dissipators . . . 100

5.3.2 Compare Performance: PID, Lag-Stabilized, Fractional . 107

5.4 Optimal Control . . . . . . . . . . . . . . . . . . . . 112

5.5 Robust/Tracking Control . . . . . . . . . . . . . . . . . . 116

5.6 Adaptive/Tracking Control . . . . . . . . . . . . . . . . . . 119

5.7 Chapter Summary . . . . . . . . . . . . . . . . . . . . . 122

\section{Part II Applications: Case Studies}

6 Case Study \#1: Control Design Issues . . . . . . . . . . . . . . 127

6.1 Introduction . . . . . . . . . . . . . . . . . . . 127

6.2 Nonlinear Second-Order System with Sinusoidal Damping . . . . 127

6.3 An Extension of Eigenanalysis to MIMO Nonlinear Systems . . . 132

6.4 Two-Mass Numerical Example . . . . . . . . . . . . . . . . . 135

6.4.1 Linear MIMO System Controller Design . . . . . . . . . . 136

6.4.2 Nonlinear MIMO System Controller Design . . . . . . . . 144

6.5 Noncollocated Control . . . . . . . . . . . . . . . . . . . . . 152

6.6 Chapter Summary . . . . . . . . . . . . . . . . . . . . . . 160 
7 Case Study \#2: Collective Systems and Controls . . . . . . . . . . . 161

7.1 Introduction . . . . . . . . . . . . . . . . . . 161

7.2 Equilibrium Collective Systems . . . . . . . . . . . . . . 163

7.3 Kinematic Collective Control . . . . . . . . . . . . . . . . . 173

7.3.1 Kinematic Control Design . . . . . . . . . . . . . . . . . 173

7.3.2 Robot Description . . . . . . . . . . . . . . . . . 175

7.3.3 Information Flow: A Trade-off Between Processing,

Memory, and Communications . . . . . . . . . . . 176

7.4 Kinetic Collective Control . . . . . . . . . . . . . . . . . . . . 177

7.5 Fisher Information and Equivalency . . . . . . . . . . . . . . . . 179

7.6 Chapter Summary . . . . . . . . . . . . . . . . . . . . . . . . . 184

8 Case Study \#3: Nonlinear Aeroelasticity . . . . . . . . . . . . . 185

8.1 Introduction . . . . . . . . . . . . . . . . . . 185

8.2 Nonlinear Stall Flutter Model . . . . . . . . . . . . . . . . . . 187

8.2 .1 Linear Region . . . . . . . . . . . . . . . . . 188

8.2.2 Nonlinear Stall Flutter with Linear Dynamics . . . . . . . . 189

8.2.3 Nonlinear Stall Flutter with Nonlinear Dynamics . . . . . . 189

8.2.4 Controller Design . . . . . . . . . . . . . . . . . . . . 192

8.3 Specific 5 MW Wind Turbine Control Design . . . . . . . . . . . 197

8.4 Chapter Summary . . . . . . . . . . . . . . . . . . . . . . . . 202

9 Case Study \#4: Fundamental Power Engineering . . . . . . . . . 207

9.1 Introduction . . . . . . . . . . . . . . . . . . . . 207

9.2 Power Engineering Application . . . . . . . . . . . . . . . . 207

9.3 Performance of Electric Power Grid System . . . . . . . . . . . 211

9.4 Linear Adaptive Power Engineering . . . . . . . . . . . . . . . . . 214

9.5 Nonlinear Adaptive Power Engineering . . . . . . . . . . . . . . . 217

9.6 Chapter Summary . . . . . . . . . . . . . . . . . . . . . . . . 222

10 Case Study \#5: Renewable Energy Microgrid Design . . . . . . . . . 225

10.1 Introduction . . . . . . . . . . . . . . . . . 225

10.2 HSSPFC Design for a Typical OMIB System . . . . . . . . . 227

10.3 HSSPFC Applied to UPFCs and Renewable Generators . . . . . . 230

10.3.1 Example One-OMIB System with a UPFC . . . . . . . . 231

10.3.2 Example Two-Swing Equation for a Wind Turbine

Connected to an Infinite Bus Through UPFC . . . . . . . 232

10.3.3 HSSPFC Applied to UPFC and Variable Generation . . . . 233

10.3.4 Numerical Simulations . . . . . . . . . . . . . . . . . 234

10.4 Microgrid in Islanded Mode . . . . . . . . . . . . . . . . . 236

10.5 HSSPFC Applied to UPFCs for Conventional and Renewable Generators . . . . . . . . . . . . . . . . . . . . . 240

10.6 Chapter Summary . . . . . . . . . . . . . . . . . . . . . . . 243

11 Case Study \#6: Robotic Manipulator Control Design . . . . . . . . . 245

11.1 Introduction . . . . . . . . . . . . . . . . . . . 245

11.2 Evaluation of the Equations of Motion . . . . . . . . . . 246 
11.2.1 Two-Link Robot Model . . . . . . . . . . . . . . . . . . . 246

11.2.2 Evaluation of the Hamiltonian Surface Shaping . . . . . . . 248

11.2.3 Evaluation of Power Flow . . . . . . . . . . . . . . . . . 249

11.3 Tracking Controller: Perfect Parameter Matching . . . . . . . . . . 252

11.4 Chapter Summary . . . . . . . . . . . . . . . . . . . . . . . 258

12 Case Study \#7: Satellite Reorientation Control . . . . . . . . . . . 259

12.1 Introduction . . . . . . . . . . . . . . . . . . 259

12.2 Spacecraft Attitude Control Design . . . . . . . . . . . . 260

12.3 Chapter Summary . . . . . . . . . . . . . . . . . . . . . . . 267

13 Case Study \#8: Wind Turbine Control Design . . . . . . . . . . . 273

13.1 Introduction . . . . . . . . . . . . . . . 273

13.2 Wind Turbine Model . . . . . . . . . . . . . . . . . . . . . 273

13.3 Adaptive Power Flow Controller Design . . . . . . . . . . . 276

13.4 Simple Model Simulation Results . . . . . . . . . . . . . . . . . . 278

13.5 Chapter Summary . . . . . . . . . . . . . . . . . . . . . . . . 279

\section{Part III Advanced Topics}

14 Sustainability of Self-organizing Systems . . . . . . . . . . . . . . . 283

14.1 Introduction . . . . . . . . . . . . . . . . . . 283

14.2 Simple Nonlinear Satellite System _ . . . . . . . . . . . 286

14.2.1 Conservation Equations for the Engine Component (Control Volume 1) . . . . . . . . . . . . . . . 287

14.2.2 Conservation Equations for the Machine (Control Volume 2) . . . . . . . . . . . . . . . . . . . 289

14.2.3 Conservation Equations for the Total System (Control Volume 12) . . . . . . . . . . . . . . . . . 291

14.3 Lifestyle Definition . . . . . . . . . . . . . . . . . . 292

14.3.1 Deformation of Potential Field with Information Flow . . . 294

14.4 Exergy Sustainability: An Energy Surety Approach _ . . . . . . 296

14.4.1 Optimality . . . . . . . . . . . . . . . . . . . 297

14.4.2 Scalability . . . . . . . . . . . . . . . . 303

14.5 Chapter Summary . . . . . . . . . . . . . . . . . . . 304

References . . . . . . . . . . . . . . . . . . 307

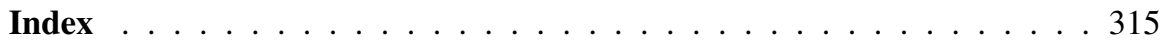

